\title{
4D model of hemodynamics in the abdominal aorta
}

\author{
Ireneusz Zbicinski ${ }^{\mathrm{a},}{ }^{\text {, }}$, Natalia Veshkina ${ }^{\mathrm{a}}$ and Ludomir Stefańczyk ${ }^{\mathrm{b}}$ \\ ${ }^{a}$ Faculty of Process and Environmental Engineering, Lodz University of Technology, \\ 213 Wólczańska St., 90-924 Lodz, Poland \\ ${ }^{b}$ Department of Radiology and Diagnostic Imaging Medical University of Łódź, 22 Kopcińskiego Str., \\ 90-153 Lodz, Poland
}

\begin{abstract}
The paper presents an application of the FSI technique to determine hemodynamics in the abdominal aorta (AA). To establish boundary conditions for the FSI study, MR anatomical data and 4D MRI velocity-mapping data (in three blood flow velocity directions and time) were collected to acquire realistic geometry of the AA and blood velocity. The mechanical parameters of the patient-specific aortic wall were applied in FSI simulations to describe wall mechanics and blood flow in the AA. Comparison of calculated and measured blood flow patterns and flow rate waveforms shows good agreement, which proves that wall pulsations should be incorporated into simulations that determine hemodynamics in the AA. The results of this work suggest that FSI analysis based on patient-specific data, such as the mechanical parameters of the aortic wall, real geometry of the aorta, and 4D flow information, might be used to predict the development of cardiovascular diseases.
\end{abstract}

Keywords: FSI-CFD, abdominal aorta, MRI velocity mapping

\section{Introduction}

The development of Computational Fluid Dynamics (CFD) was a great contribution to the prediction of blood flow behavior in blood vessels. The CFD technique can be used to gain information about the magnitude of wall shear stress and pressure distribution on the arterial walls, which is difficult to measure in vivo but is essential to predict the development of some cardiovascular diseases, such as stenosis or aneurysms $[1,2]$.

In order to analyze the flow pattern and shear stresses occurring inside the aorta, numerical studies of the aorta have been performed in the past for CFD models, and structural models that show the influence of geometry and composition on the stress pattern of the aortic wall have been developed.

The most recent studies combine fluid dynamics with structural analysis, using Fluid-Structure Interaction (FSI) analysis [3, 4]. The FSI technique is used to describe a mutual response of the solid and fluid phases simultaneously and is widely used to assess the conditions of the cardiovascular system, namely the interaction between the vessel wall and the blood flowing inside. Pressure produced by blood flow shifts the vessel wall, while deformation of the vessel wall affects the flow

\footnotetext{
* Address for correspondence: Ireneusz Zbicinski, Faculty of Process and Environmental Engineering, Lodz University of Technology, 213 Wólczańska St., 90-924 Lodz, Poland. Tel.: +48 42 6313773; Fax: +48426365663; E-mail: ireneusz.zbicinski@.p.lodz.pl.
} 
structure inside the vessel [5].

The mechanical behavior of arterial walls has been a subject of research for over 100 years, with the first report from Charles S. Roy [6], who analyzed the elastic properties of the vessel wall. As vascular diseases are associated with changes in the mechanical properties of the arterial walls, characterizing and studying their biomechanical behavior might allow the prediction of risk development for some cardiovascular diseases [7].

The FSI method is based on the execution of simulations through a sequence of multi-field time steps, where each consists of coupling or "stagger" iterations. During every stagger iteration in each field, a solver collects data that are required from the other solver and solves field equations for the current multi-field time step. The CFD model provides total force information to the structural part, while the structural part provides the total mesh displacement information. The stagger iterations are repeated until a maximum number of stagger iterations is reached or until the data transferred between solvers have converged. The FSI simulation allows for coupling blood flow physics and aortic wall physics throughout the solution process. Although the FSI technique requires excessive computation time and vast memory requirements, the simulation results have better agreement with realistic blood flow predictions than the CFD or structural models $[8,9]$.

The main aim of this work was to develop and verify the FSI model of hemodynamics in the abdominal aorta.

\section{Methods}

\subsection{Patient-specific model reconstruction}

In the first step of this work, patient specific geometry was determined from Magnetic Resonance Imaging (MRI). The geometry of the human aorta was reconstructed for a male volunteer with no history of vascular diseases. MRI data for geometry reconstruction were acquired in Hammersmith Hospital, in the Robert Steiner MR Unit, with assistance from the Imperial College of London, UK. The MR 554 slices were collected with the following parameters: repetition time of $3.2 \mathrm{~ms}$, echo time of $1.6 \mathrm{~ms}$, interslice distance of $5 \mathrm{~mm}$, and image frequency of $63.8 \mathrm{MHz}$. The images were acquired as a set of $2 \mathrm{D}$ axial images throughout the length of the aorta; these contained information about the geometry of the object and blood velocity.

\subsection{Calculations of the fluid part}

The segmentation of all acquired images and 3D geometry reconstruction were accomplished using Materialise's Interactive Medical Image Control System (MIMICS 14.0). In the next step, the 3D object of the aorta was imported into a mesh generation package, ANSYS ICEM CFD (ICEM Engineering, Warwick CV34, UK), from which the external surface of the geometry was reconstructed.

To reduce convergence problems, a fine mesh was developed near the wall, with the height of the wall boundary elements having $y+$ less than 2 and a maximum of at least 10 grid nodes inside the boundary layer $(y+$ is a nondimensional variable based on the distance from the wall, through the boundary layer, and to the first node away from the wall). Following the exponential law of increasing height, ANSYS ICEM CFD automatically sized the layers by specifying the growth factor and the height of the first layer nearest the wall. Finally, a mesh size of 234,166 nodes was used for 


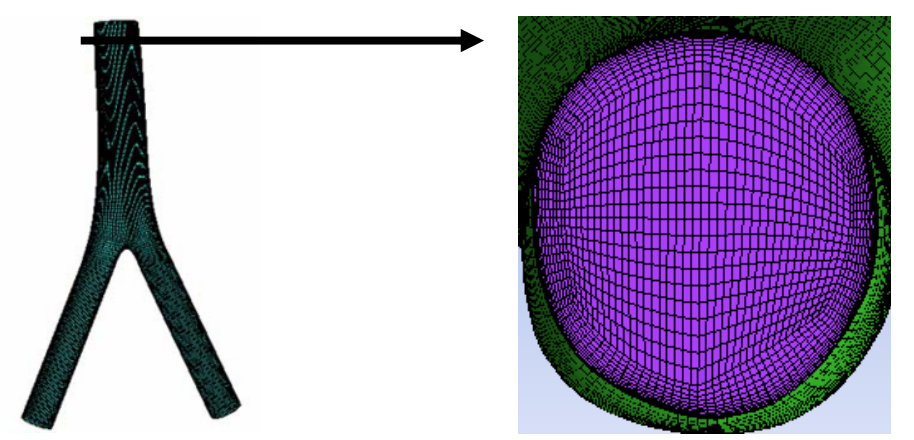

Fig. 1. Structured hexahedral mesh generated for the fluid part with ICEM CFD.
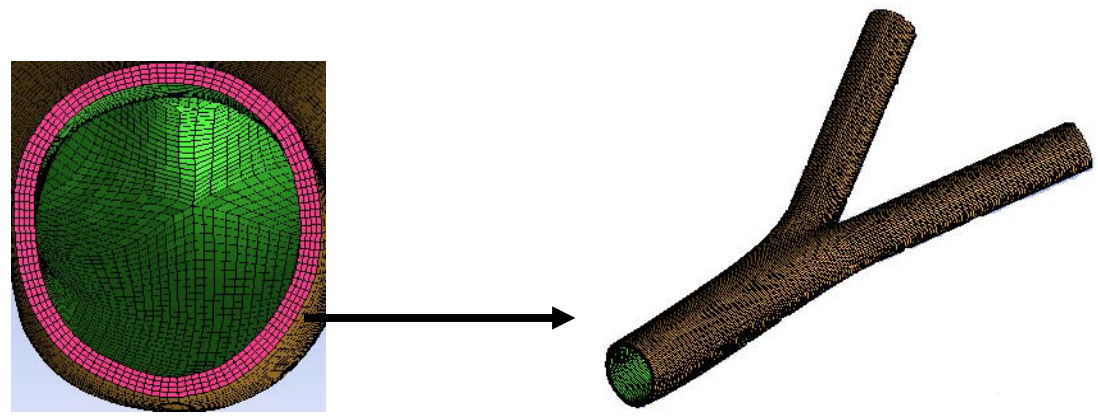

Fig. 2. Hexahedral mesh for the aortic wall generated by ICEM CFD.

the calculations. Figure 1 shows the hexahedral structured mesh in the fluid part, which was generated fluid part of the model for the reconstructed AA geometry.

Blood flow was modeled as a homogeneous, incompressible, Newtonian fluid with a density of $1060 \mathrm{~kg} / \mathrm{m}^{3}$ and a viscosity of 3.4E-3 Pas. The turbulence level at the inlet was set to $1.5 \%$. No-slip boundary conditions were applied on the aortic wall [10]. The patient pressure in the iliac arteries was reconstructed from the pressure waveform determined by [1].

\subsection{Calculations of the solid part of the aorta}

The wall of the aorta was modeled as an incompressible, homogeneous, isotropic, hyperelastic material. To calculate the displacement of the arterial wall, we used the Yeoh hyperelastic model [11]:

$$
\mathrm{W}=\alpha\left(\mathrm{I}_{\mathrm{B}}-3\right)+\beta\left(\mathrm{II}_{\mathrm{B}}-3\right)^{2}
$$

where $\mathrm{W}$ is the strain energy density function, $\mathrm{I}_{\mathrm{B}}$ and $\mathrm{II}_{\mathrm{B}}$ are the first and second invariants of $\mathrm{B}$, and $\mathrm{B}$ is the Left Cauchy-Green tensor. The material constants of the aortic wall $\alpha=14.25 \mathrm{~N} / \mathrm{cm}^{2}$ and $\beta=11.4$ $\mathrm{N} / \mathrm{cm}^{2}$ were determined earlier [12]. The aortic wall was meshed with 86,664 hexahedral elements, with a maximum edge length of $0.2-0.3 \mathrm{~mm}$. Figure 2 shows the hexahedral mesh for the aortic wall applied in the calculations.

The fluid and solid domains were coupled using ANSYS 14.1 commercial software.

\section{Results and discussion}


Analysis of the results includes a comparison of simulated velocity profile contours with MRImeasured velocity data, as well as a comparison of the flow rates of the simulated and measured flows in the AA [12].

\section{1. $3 D$ velocity profiles}

In order to provide patient-specific flow conditions in the AA, a 4D Phase - Contrast (PC) MRI velocity-mapping technique was used to acquire the flow images. The $4 \mathrm{D}$ blood flow data set contained information on 3D blood flow in three orthogonal directions throughout the cardiac cycle. The 4D PC MRI sequences enabled the acquisition of flow data that are linearly related to the actual blood flow velocities, capturing both speed and direction.

Analysis of the results includes a comparison of simulated velocity profile contours with MRI measured velocity data, as well as a comparison of the flow rates between the simulated and measured flows. Figure 3 shows the MRI velocity measured below the inlet region and the axial velocity contours from the flow simulation at the same location. The comparison was made in four different time points during a cardiac cycle. We can see that the velocity profiles are in good quantitative and qualitative agreement in every analyzed point (mid-systolic acceleration, peak systole, late systole deceleration, and diastole). The maximum difference between the measured and calculated velocities

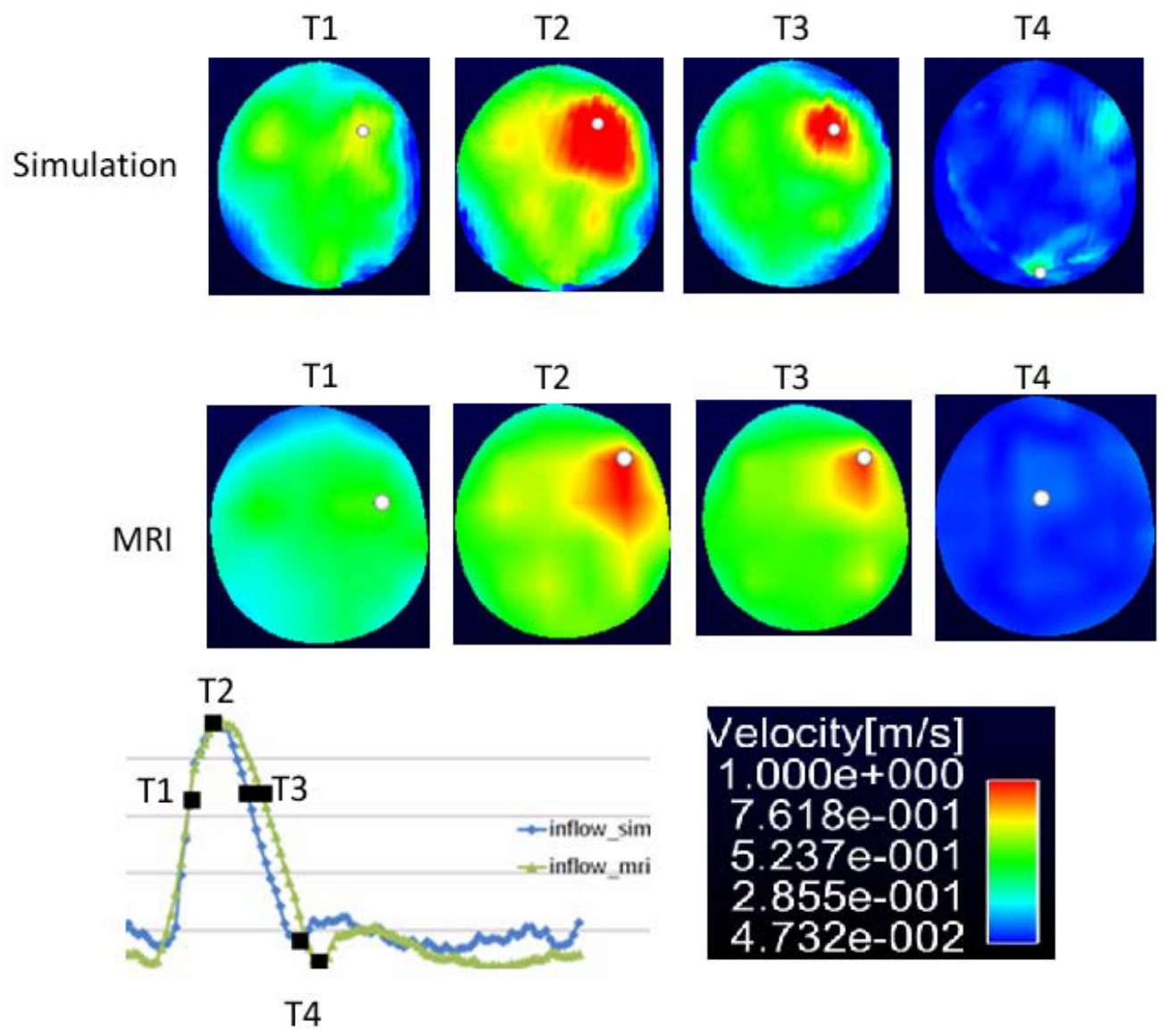

Fig. 3. Comparison of the MRI velocity at the inlet region with axial velocity contours from flow simulation. 
is found in the diastole phase and is equal to about $5 \%$. Comparison of the boundary layer region in the MRI measurements and the simulation results shows that the boundary layer is not clearly visible in the MRI data. The absence of the boundary layer region in MRI measurements is one of the drawbacks of this technique. The lack of a boundary layer region can be explained by the partial volume effect in MR velocity measurements (when a pixel represents more than one kind of tissue type); therefore, near the wall, velocity values are subject to some uncertainties.

This drawback can be explained by the fact that MR phase contrast imaging normally has a very low signal-to-noise ratio in the regions where flow is slow, such as near the wall region and at time phases where the flow reverses its direction. In such regions, most of the spins have near-zero velocities, so accurate velocity image segmentation becomes very difficult in this region. In the simulation results (Figure 3), a low velocity is clearly presented in the boundary layer region that corresponds to the normal physiological conditions.

Figure 4 shows a comparison of the predicted velocity distribution with MRI measurements along the axial direction. It displays the variation in simulated velocity in the axial direction and the measured velocity during midway flow acceleration, the peak systole, midway flow deceleration, and during the maximum reverse flow phase. We can observe that the distribution of velocities is in

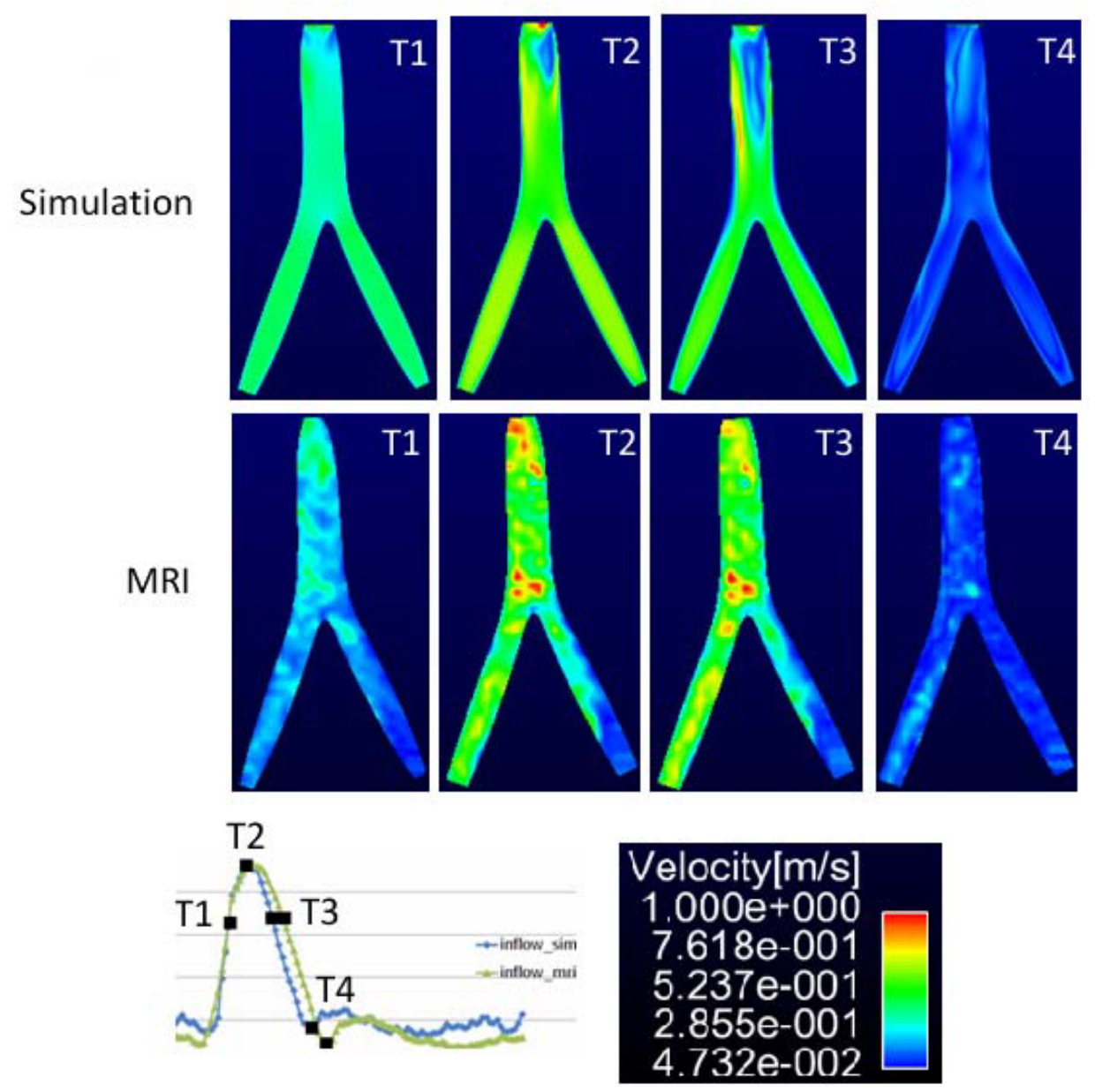

Fig. 4. Velocity distribution along the axial direction. 
qualitative agreement between the simulated and measured data. From the MRI measurements, we can see that at points $\mathrm{T} 2$ and $\mathrm{T} 3$, the velocity is higher in the left iliac artery than in the right iliac artery, while the simulation results show similar velocity profiles in both iliac arteries. Taking into account that the MR images were acquired from a young healthy volunteer with no previous history of vascular diseases, the flow pattern in both iliac arteries should be congenial. Uncertainties from MRI measurements might be caused by mismatching the geometry of the aorta with collected 4D velocity data and extracting the velocity profile from a full set of 4D MRI data. Therefore, it might be useful for diagnostic purposes to treat the simulation results as a reliable tool for investigating abnormal cases.

$$
\mathrm{T} 1=0.04 \mathrm{~s} \quad \mathrm{~T} 2=0.13 \mathrm{~s} \quad \mathrm{~T} 3=0.20 \mathrm{~s} \quad \mathrm{~T} 4=0.79 \mathrm{~s}
$$
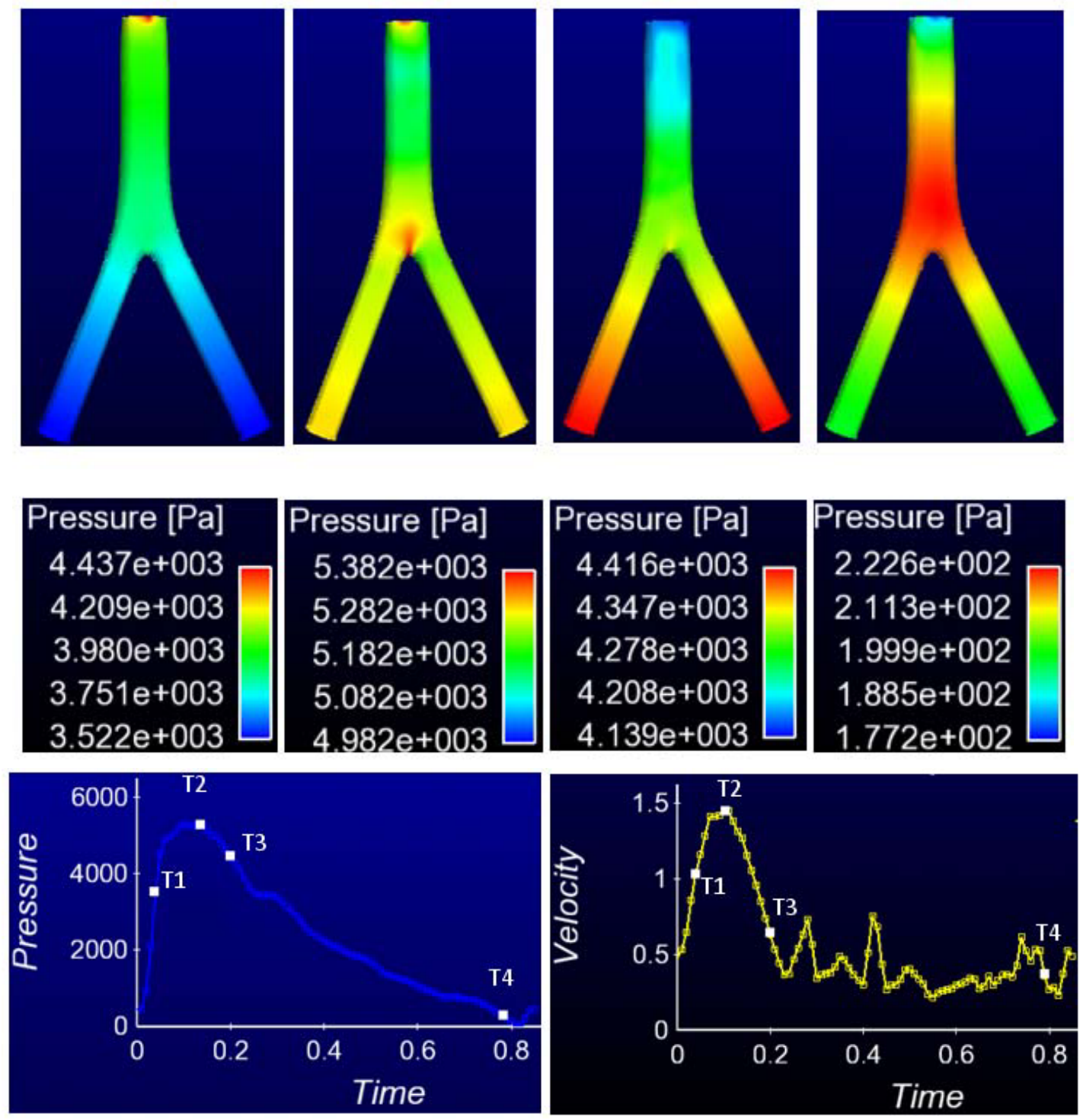

Fig. 5. Pressure distribution at different time points during the cardiac cycle. 


\section{2. $3 D$ pressure profiles}

Figure 5 shows the pressure distribution on the arterial wall at four different time points of the cardiac cycle. At point T1, which corresponds to the mid-acceleration phase, we observe that pressure falls from inlet to exit, as the total pressure decreases due to flow friction. Simultaneously during the acceleration phase, velocity falls from inlet to exit, which is confirmed by the positive pressure gradient observed in T1. Point T2 corresponds to the peak systole - there is zero acceleration or retardation, which agrees with a pressure gradient that is close to zero. During mid-systolic deceleration (point T3), velocity falls - a negative pressure gradient is observed. During diastole (point T4), the maximum pressure is observed in the bifurcation zone, which corresponds to the location of the maximum displacement of the aortic wall during the cardiac cycle.

The presence of the spot of high pressure observed near the inlet region during mid-acceleration and peak systole can be explained by a specific velocity profile, applied as a boundary condition.

\section{Conclusions}

The FSI technique was employed to analyze flow and pressure patterns in the AA with the application of patient-specific mechanical parameters of the aortic wall.

Excellent agreement between hemodynamics in the aorta determined by the 4D FSI model and MRI measurements proves that the pulsations of the aortic wall should be incorporated into calculations to capture the complex flow structure in the AA.

Since hemodynamic factors play an important role in evaluating the development of cardiovascular diseases, MRI data can provide only limited information on the local hemodynamics. Therefore, combined MR and FSI analysis can be used to explore and predict the development of cardiovascular diseases. The verified FSI model of blood flow in the AA can be used to supply more flow detail than MRI or CT-USG measurements alone.

\section{Acknowledgments}

This work was supported by the National Science Centre of Poland (grant number 2011/01/B/ST8/06689). The authors thank Professor X.Y. Xu and Professor N.B. Wood from Imperial College London for helpful discussion and excellent guidance.

\section{References}

[1] M.S. Olufsen and C.S. Peskin, Numerical simulation and experimental validation of blood flow in arteries with structured-tree outflow conditions, Biomedical Engineering 28 (2000), 1281-1299.

[2] W. Lee, Biofluid Mechanics in Cardiovascular Systems, The McGraw-Hill Companies, Inc., 2006.

[3] F.P.P. Tan, R. Torii, A. Borghi, R.H Mohiaddin, N.B. Wood and X.Y. Xu, Fluid-structure interaction analysis of wall stress and flow patterns in a thoracic aortic aneurysm, International Journal of Applied Mechanics 1 (2009), 179-199.

[4] A. de Vecchi, D.A. Nordsletten, R. Razavi, G. Greil, N. P. Smith, Patient specific fluid-structure ventricular modelling for integrated cardiac care, Medical and Biological Engineering and Computing 51 (2013), 1261-1270.

[5] D.S. Molony S. Broderick, A. Callanan, T.M. McGloughlin and M.T. Walsh, Fluid-structure interaction in healthy, diseased and endovascularly treated abdominal aortic aneurysms, biomechanics and mechanobiology of aneurysms studies in mechanobiology, Tissue Engineering and Biomaterials 7 (2011), 163-179. 
[6] C.S. Roy, The elastic properties of the arterial wall, Journal of Physiology 3 (1881), 125-159.

[7] A. Duprey, Mechanical properties of the aorta, Annual Report to the European Society for Vascular Surgery 2007-2008.

[8] J.R. Cebral, P.J. Yim, et al. Blood flow modeling in carotid arteries with computational fluid dynamics and MR Imaging, Academic Radiology 9 (2002), 1286-1299.

[9] F. Dorn, F. Niedermeyer, A. Balasso, D. Liepsch and T. Liebig, The effect of stents on intra-aneurysmal hemodynamics: In vitro evaluation of a pulsatile sidewall aneurysm using laser Doppler anemometry, Interventional Neuroradiology $\mathbf{5 3}$ (2011), 267-72.

[10]N. Veshkina, I. Zbicinski and L. Stefanczyk, 2D FSI determination of mechanical stresses on aneurismal walls, BioMedical Materials and Engineering 24 (2014), 2519-2526.

[11] M. Raghavan and D. Vorp, Toward a biomechanical tool to evaluate rupture potential of abdominal aortic aneurysm: Identification of a finite strain constitutive model and evaluation of it applicability, Journal of Biomechanics 33 (2000), $475-482$.

[12]N. Veshkina, Determination of hemodynamics in collapsible walls, PhD Dissertation, Lodz University of Technology, 2013. 